\title{
Thermodynamics of the Two-Level Richardson Model
}

\author{
A. Ciechan and K.I. Wysokiński \\ Institute of Physics and Nanotechnology Centre, M. Curie-Skłodowska University \\ Radziszewskiego 10, 20-031 Lublin, Poland
}

Dedicated to Professor Józef Spatek on the occasion of his 60th birthday

The two-level version of the Richardson model presents a unique possibility to calculate numerically exactly thermodynamic properties of the system it describes. The point is that all energies and the degeneracies of the many-body system can be easily calculated. The energies are given by the eigenvalues of the small (of order of $N \times N$, where $2 N$ is a number of electrons in the system) tridiagonal matrices. Here we numerically obtain a complete spectrum of the interacting two-level model and calculate the specific heat and the pairing energy of the small system at finite temperatures.

PACS numbers: 61.46.--w, 73.22.Gk, 74.90.+n

\section{Introduction}

The properties of materials at nanoscale differ from their equivalents in bulk. This is due to the discrete energy spectrum, parity effects and large surface to volume ratio, etc. [1-4]. Many characteristics depend on the size of the grain which may be quantified by the total number of particles in it $[5,6]$.

In a small grain the single particle spectrum consists of discrete energy levels. The spectroscopic studies of such system always find the gap in the excitations spectrum. In the noninteracting case it is of the order of mean value of the single particle level distance $d$. If the system enters the superconducting state, the excitation energy increases from its normal state value $\sim d$ to the value $\sim \Delta$, which is larger [7] than the bulk superconducting gap $\Delta_{\mathrm{b}}$. Obviously for ultrasmall grains, for which $d>\Delta_{\mathrm{b}}$ the normal contribution will dominate [8] and it is very difficult to unambiguously detect the signatures of superconductivity. In this limit it has been proposed to study a magnetic response [9] and the spectral function for the Cooper pair transfer [10] as these parameters behave in a different way in normal and superconducting grains.

The temperature dependence of the specific heat has always played an important role in identifying the phase transition. It is thus of interest to study this 
parameter in the context of very small grains with pairing interactions. In the present work we calculate numerically exact energies of the $N$ electron pairs in two highly degenerate levels. The knowledge of their degeneracies allows an easy calculation of average energy, specific heat, and pairing energy of the system.

In Sect. 2 we present the model and its solution. The results of numerical calculations for very small grains have been discussed in Sect. 3. We summarize our main findings in Sect. 4.

\section{The model and the solution}

Let us consider a small system with two highly degenerate energy levels close to the Fermi energy with $2 N$ or $2 N+1$ electrons interacting by the weak attractive potential. We model it by the following Hamiltonian of the reduced BCS form [1]:

$$
H=\sum_{f, \sigma= \pm} \epsilon_{f} c_{f \sigma}^{+} c_{f \sigma}-g \sum_{f, f^{\prime} \in S} c_{f+}^{+} c_{f-}^{+} c_{f^{\prime}-} c_{f^{\prime}+},
$$

where $(f \sigma)$ denote the single particle quantum numbers, $\epsilon_{f}=\epsilon_{0}, \epsilon_{1}$ are the single electron energy levels $\left(\epsilon_{1}-\epsilon_{0}=h\right), g$ is the positive coupling constant, $c_{f \sigma}^{+}, c_{f \sigma}$ are fermion creation and annihilation operators.

The model (1) has been widely used in the study of superconductivity in small metallic grains [1]. Its mean field BCS solution obtained within the grand canonical ensemble relies on the ground state wave function which does not conserve a particle number and thus is not suitable for the description of a small system with a finite number of particles and large energy fluctuations. However, it turns out that the model (1) has an exact solution. This has been shown in the early sixties by Richardson [11]. For $\epsilon_{0(1)}=\mp h / 2$ the energy $E$ of the grain containing exactly $N$ pairs is given by the solution of the following system of $(N+1)$ algebraic equations

$$
\left(\omega_{\mu}-E\right) \varphi(\mu)-A_{\mu} \varphi(\mu+1)-B_{\mu} \varphi(\mu-1)=0,
$$

where the parameter $\mu=N_{2}-N_{1}(\mu=-N,-N+2+\cdots, N)$ gives the difference between a number of pairs in upper and lower energy level and $\varphi(\mu)$ is the corresponding eigenfunction. The other parameters are defined as

$$
\begin{aligned}
& \omega_{\mu}=\mu h-g(N-\mu)\left(\Omega_{0}-N+\mu+1\right)-g \mu\left(\Omega_{1}-\mu+1\right), \\
& A_{\mu}=g(N-\mu)\left(\Omega_{1}-\mu\right), \\
& B_{\mu}=g \mu\left(\Omega_{0}-N+\mu\right) .
\end{aligned}
$$

Here $\Omega_{0(1)}$ denote the pair degeneracies of the level $0(1)$. Solving these equations one obtains $(N+1)$ roots for the energy $E$ and the corresponding wave functions $\varphi(\mu)$. The lowest of the roots is the ground state energy of the system, the others are excited states.

For the numerical purposes we measure all energies in units of $h=1$. Equations (2)-(5) with $N$ pairs distributed among two levels give the lowest energies. To obtain other energies [12] of the system one breaks a single pair and solves the system of equations for $(N-1)$ pairs and two free electrons. These two electrons 
(from the broken pair) block two single particle states which do not take part in the pair scattering process induced by the second term in Eq. (1). Their energy is added to the energy of the system [12]. This process has to be continued until all pairs are broken.

The total energy of $N_{\mathrm{p}}$ pairs and $N_{\mathrm{b}}$ electrons out of which $\mu$ pairs and $i_{\mathrm{b}}$ electrons occupy the upper level is given by

$$
E\left(N_{\mathrm{p}}-\mu, \mu, N_{\mathrm{b}}-i_{\mathrm{b}}, i_{\mathrm{b}}\right)=E_{\mu}+\left(N_{\mathrm{b}}-i_{\mathrm{b}}\right) \varepsilon_{0}+i_{\mathrm{b}} \varepsilon_{1},
$$

where $E_{\mu}, \mu=0,1, \ldots N_{\mathrm{p}}$ are the solutions of Eq. (2). The important issue is the degeneracy of each energy eigenvalue. For given degeneracies of the levels $\Omega_{0}$ and $\Omega_{1}$ and given quantum numbers $I\left(I=\left\{N_{\mathrm{p}}, \mu, N_{\mathrm{b}}, i_{\mathrm{b}}\right\}\right)$ we get from Eq. (6) a precise energy eigenvalue $E_{I}$ and assign to it the degeneracy factor $D_{I}$. For the two level system $D_{I}$ is given by

$$
D_{I}=2^{N_{\mathrm{b}}}\left(\begin{array}{c}
\Omega_{0} \\
N_{\mathrm{p}}-\mu \Omega_{0}-\mathrm{N}_{\mathrm{p}}+\mu \\
N_{\mathrm{b}}-i_{\mathrm{b}}
\end{array}\right)\left(\begin{array}{c}
\Omega_{1} \\
\mu
\end{array}\right)\left(\begin{array}{c}
\Omega_{1}-\mu \\
i_{\mathrm{b}}
\end{array}\right) .
$$

In Fig. 1 we show the dependence of the energies $E_{i}, i=0,1, \ldots, N$ on the parameter $g$. These are the solutions of Eqs. (2)-(5) for a small system with $N=10$ pairs, the pair degeneracy [12] of levels $\Omega_{0}=\Omega_{1}=N$ and no broken pairs.

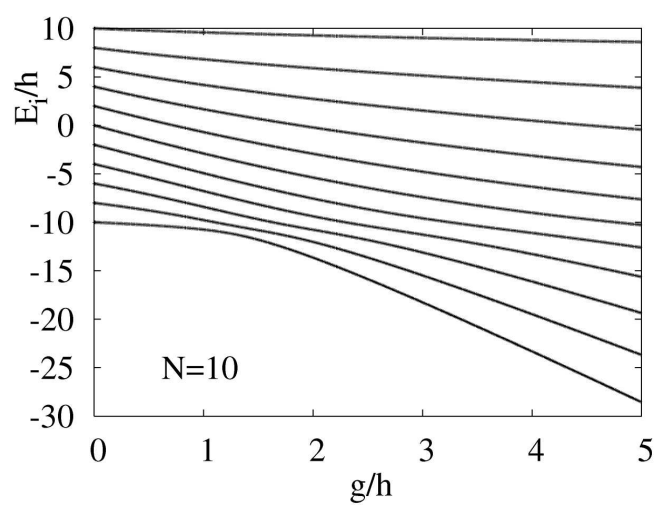

Fig. 1. The $g$ dependence of the energies $E_{i}$ being solutions of Eqs. (2)-(5) for $N=10$ pairs. All energies are measured in units of inter-level distance $h$.

Let us note the strong dependence of the ground state energy on $g$ and much weaker dependence of higher energies.

\section{Thermodynamics}

The two-level model of the superconducting grain studied here presents a rare example of the interacting many-body system for which we can easily and numerically exactly calculate all energies $E_{I}$ and their degeneracies $D_{I}$. This allows the calculation of the thermal averages within canonical ensemble. Here we focus 
on small systems containing up to $N=50$ pairs. The pair degeneracy of the levels is taken to be $\Omega_{0}=\Omega_{1}=2 N$. We study systems containing even and odd number of electrons. In the even case one of the single particle states remains blocked by an unpaired electron and is not accessible to pair scattering. At zero temperature the extra electron occupies the lower energy level, but at higher $T$ it may be excited to other levels as also the pairs are.

We first calculate the temperature dependent pairing energy $E_{\mathrm{p}}$, defined as a difference between average energy of the interacting and non-interacting systems $[8]$

$$
E_{\mathrm{p}}(g, T)=\langle E(g)\rangle-\langle E(g=0)\rangle,
$$

where $\langle E\rangle=\frac{1}{Z} \sum_{I} D_{I} E_{I} \exp \left(-E_{I} / k T\right)$ is the thermal average of the energy, $Z=\sum_{I} D_{I} \exp \left(-E_{I} / k T\right)$ is the canonical partition function, and $k$ is the Boltzmann constant. The knowledge of temperature dependence of the average (internal) energy $\langle E\rangle$ allows an easy calculation of the heat capacity of the system via

$$
C_{V}(T)=\left(\frac{\partial\langle E\rangle}{\partial T}\right)_{N, V}
$$

Figure 2 shows the temperature dependence of the pairing energy of the ultrasmall grain containing 20 electrons (10 pairs) or 21 electrons (i.e. 10 pairs and an extra electron, which at low temperatures occupies a state in the lower level). At very low temperatures pairing energy is nearly $T$-independent. It starts

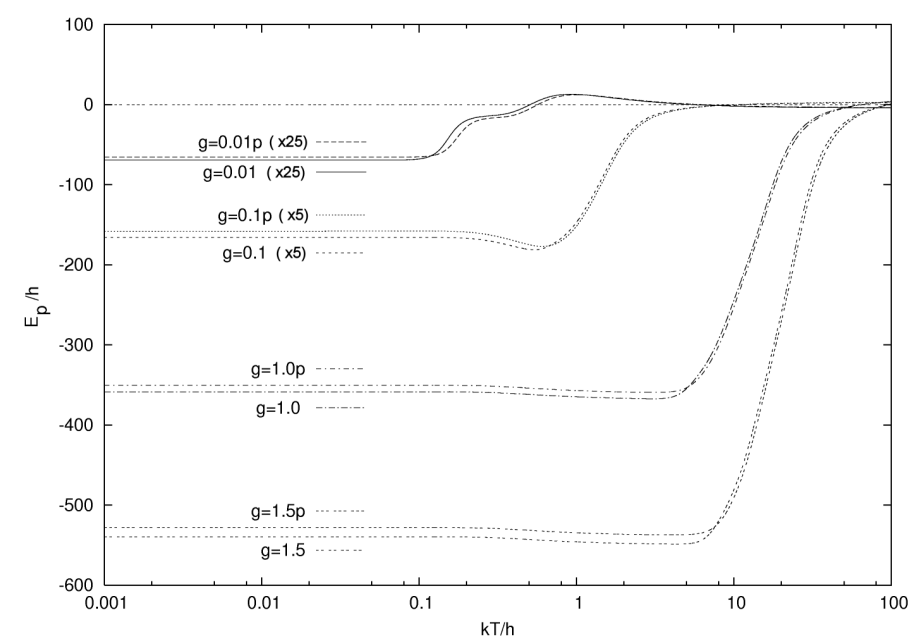

Fig. 2. The temperature dependence of the pairing energies of the grain containing 20 or 21 electrons ( $N=10$ pairs plus 0 or 1 electron) for a few values of $g=0.01,0.1,1.0,1.5$ (from top to bottom). The curves corresponding to $g=0.01$ have been multiplied by 25 , and those to $g=0.1$ by 5 . In each group the lower curve corresponds to a system with an even number of electrons. The symbol "p" next to values of $g$ denotes an extra particle in an odd system. 


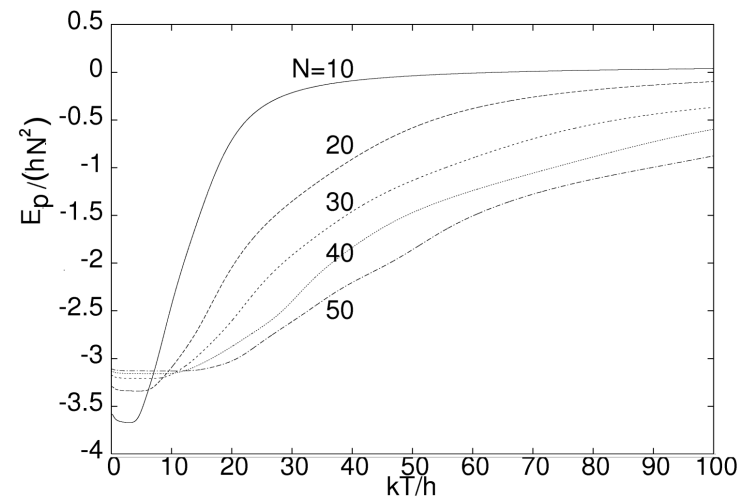

Fig. 3. The temperature dependence of the pairing energies of the grains characterized by the constant coupling $g=1.0 h$ for $N$ pairs with $N=10,20,30,40,50$ (from top to bottom). The normalization of the calculated pairing energies have been obtained by dividing the bare energy by $N^{2}$. It is interesting to note that at lowest temperatures normalized pairing energy is lowest for the smallest grains, while at elevated temperatures the opposite is true.

to increase at characteristic temperature, which for a given number of pairs in the system depends on coupling constant $g$. It is thus natural to consider this temperature as a characteristic transition temperature in a finite system. Systems containing an even number of pairs have slightly lower (negative) pairing energy. It means that they are more strongly bound. It is interesting to note that the normalized pairing energy, i.e. $E_{\mathrm{p}} / N^{2}$, calculated for the constant coupling $g$ has (at low temperatures) the largest (negative) value for smallest systems and decreases for larger systems. The dependence of normalized pairing energy on temperature for $g=1.0 h$ and different values of $N$ is shown in Fig. 3. At higher temperatures the pairing energy per particle is larger (as to the modulus) for larger $N$.

It is important to realize that because the pairing energy (for a given $N$ ) strongly depends on the coupling $g$ (Fig. 2) the dependence of $E_{\mathrm{p}}$ on $T$ may be different than the one observed here if one takes into account the changes of $g$ with grain size (or $N$ ) as postulated in Ref. [13].

It is well known that the heat capacity of the two-level non-interacting system is dominated [14] by the Schottky anomaly. In Fig. 4 we show the heat capacity for an interacting two-level system described by the Hamiltonian (1). If the coupling $g$ is very small $<0.1$ one observes two characteristic features of $C_{V}$. First is the contribution of the normal electrons which appear in the system as a result of temperature induced pair breaking. This contribution is visible as a peak centred around $k T / h \approx 0.5$. The other characteristic feature is the well-developed and strongly $g$ dependent peak at very low temperatures. It is connected with collective fluctuations in the systems. In our case it is mainly due to intra- (and to less 

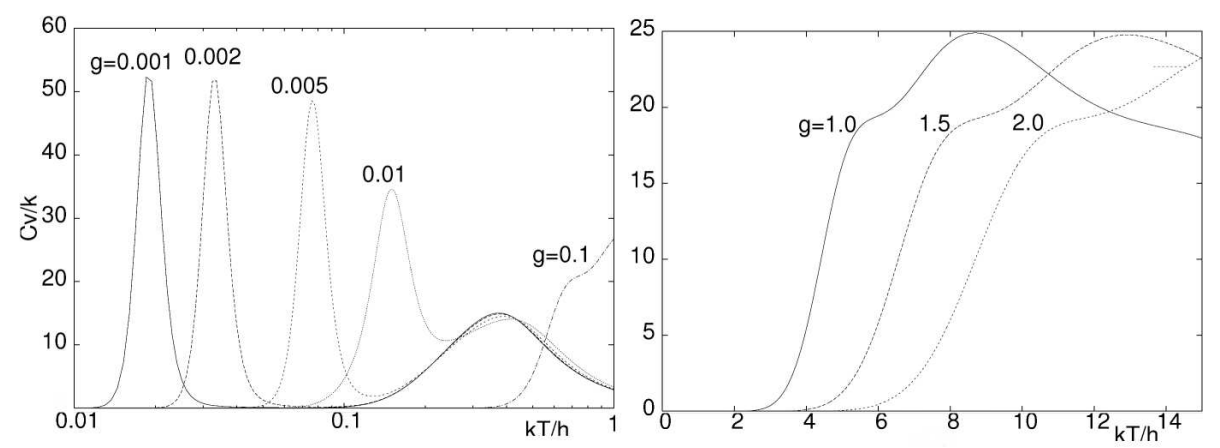

Fig. 4. The heat capacity for ultra small grain with $N=10$ pairs for lower than 1 (left part) and larger than 1 values of $g$ (right part). Each curve is marked by the actual $g$-value.

extent) inter-level pair excitations and pair breaking. With increasing $g$ this peak moves to higher temperatures merging with the main peak. Already for $g=0.1$ collective peak disappears and only a shoulder is visible on the left side of the main peak (see left part of Fig. 4). The precise identification of various contributions to the energy fluctuations requires an additional discussion and is outside the scope of the present paper. Here we merely note that the characteristic shoulder on the $C_{V}(T)$ curves is present also for higher values of $g$ as can be seen in the right part of Fig. 4.

\section{Summary and discussion}

On the basis of the exact solution of the interacting model we have calculated the specific heat and superconducting signature (pairing energy) of the two-level model. For very small values of $g$ there are clearly separated two contributions to the heat capacity: one is of collective character and is present at very low temperatures while the other is essentially identical to that of the non-interacting particles (known as the Schottky peak in the two-level systems). For larger values of $g$ the collective branch merges with the single particle one. In larger systems we also observe the remnants of it as the shoulder on the low $T$ specific heat.

The parity effects have been observed both in the pairing energy and specific heat and found to persist up to temperatures $k T / h$ of order 2 or higher. The grains with an even number of particles have a larger pairing energy than those containing an odd number of particles.

\section{Acknowledgments}

This work was partially supported by the State Committee for Scientific Research grant no. 2P03B 06225. 


\section{References}

[1] J. von Delft, D.C. Ralph, Phys. Rep. 345, 61 (2001).

[2] K.A. Matveev, A.J. Larkin, Phys. Rev. Lett. 78, 3749 (1997).

[3] E.A. Yuzbashyan, A.A. Baytin, B.L. Altshuler, Phys. Rev. B 71, 094505 (2005).

[4] J. Dukelsky, S. Pittel, G. Sierra, Rev. Mod. Phys. 76, 643 (2004).

[5] A. Ciechan, K.I. Wysokiński, Acta Phys. Pol. A 109, 569 (2006).

[6] S. Dusuel, J. Vidal, Phys. Rev. A 71, 060304 (2005).

[7] D.C. Ralph, C.T. Black, M. Tinkham, Phys. Rev. Lett. 78, 4087 (1997); C.T. Black, D.C. Ralph, M. Tinkham, ibid. 74, 3241 (1995); ibid. 76, 688 (1996).

[8] M. Schechter, J. von Delft, Y. Imry, Y. Levinson, Phys. Rev. B 67, 064506 (2003).

[9] V.N. Gladilin, V.M. Fomin, J.T. Devreese, Phys. Rev. B 70, 144506 (2004).

[10] R. Rossignoli, N. Canosa, J.L. Egido, Phys. Rev. B 64, 224511 (2001).

[11] R.W. Richardson, Phys. Lett. 3, 277 (1963); Phys. Lett. 5, 82 (1963); Phys. Lett. 14, 325 (1965); J. Math. Phys. 6, 1034 (1965); Phys. Rev. 141, 949 (1966); Phys. Rev. 144, 874 (1966); Phys. Rev. 159, 792 (1967); J. Math. Phys. 18, 1802 (1977).

[12] R.W. Richardson, N. Sherman, Nucl. Phys. 52, 221 (1964).

[13] K. Van Houcke, S.M.A. Rombouts, L. Pollet, Phys. Rev. B 73, 132509 (2006).

[14] R. Kubo, Statistical Mechanics, North-Holland Publ. Co., Amsterdam 1965, p. 38. 